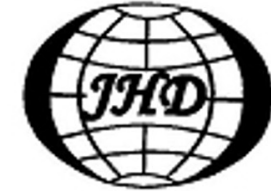

www.sciencedirect.com/ science/journal/10016058

\title{
Observation of the induced pressure in a hybrid micro/nano-channel
}

\author{
KONG Gao-pan (孔高攀), ZHENG Xu (郑旭), SILBER-LI Zhan-hua (李战华) \\ The State Key Laboratory of Nonlinear Mechanics, Institute of Mechanics, Chinese Academy of Sciences, Beijing \\ 100190, China, E-mail: kun88d@gmail.com \\ XU Zheng (徐征) \\ Key Laboratory for Micro/Nano Technology and System of Liaoning Province, Dalian University of Technology, \\ 116024 Dalian, China
}

(Received June 24, 2012, Revised September 29, 2012)

\begin{abstract}
This paper studies the flow characteristics in micro/nano-channels subjected to an applied electric field. The nano-channel flow was observed by means of the fluorescence Calcein. A Fluorescence Concentration Gradient Interface (FCGI) was observed across the nano-channel array. The front of the FCGI was shown to have an analogous parabolic shape. The propagation of this interface reflects indirectly the induced pressure at the micro/nano-channel junction, where the enrichment-depletion processes are known to take place. This induced pressure was predicted by numerical simulations, and this paper gives the first experimental evidence.
\end{abstract}

Key words: micro/nano-channel, induced pressure, ion enrichment/depletion

\section{Introduction}

With the rapid development of the micro- and nano-fabrication technology, many nano-fluidic devices were developed with wide applications in laboratories. It is noted that when the characteristic length of the microfluidics channels is reduced to the same order as the Debye length, the ion selection process becomes more efficient due to the overlapping Electric Double Layers (EDL). One kind of these devices, a hybrid micro/nano-channel, was used to improve the trace element detection and its sensitivity. Wang et al. ${ }^{[1]}$ and Kim et al. ${ }^{[2]}$ observed the protein concentration enrichment (of up to a factor of 106) with this kind of system. Recently, Kim et al. ${ }^{[3]}$ developed a new and efficient device for the direct seawater desalination. The physical mechanism of these

* Project supported by the Chinese Academy of Sciences Research and Development Program of China (Grant No. KJCX2-YW-H18), the National Natural Science Foundation of China (Grant No. 10872203) and the National Key Basic Research Development Program of China(973 Program, Grant No. 2007CB714501).

Biography: KONG Gao-pan (1982-), Male, Master

Corresponding auther: SILBER-LI Zhan-hua,

E-mail: 1ili@imech.ac.cn devices is as follows.

Basically, the velocity profile of the electro-kinetic flow driven only by an applied electric potential should be shaped as a "plug". But, Kim et al. ${ }^{[4]}$ observed fast moving vortices in the depletion zone at the positively charged end of the nano-channel array in their hybrid micro/nano-channel chip. They noticed that the front of the depletion zone in the microchannel was like "meniscus" as driven by pressure. This parabolic concentration front in the microchannel was also observed and studied by $\mathrm{Yu}$ and Silber-Li ${ }^{[5]}$. These experimental results indicate that there is an induced pressure in the pure electro-kinetic flow. Jin et al. ${ }^{[6]}$ presented a numerical model based on the Navier-Stokes equation and the Nernst-Planck equation to simulate the flow in a hybrid micro/nano-channel and found that an induced pressure did exist across the micro/nano-channel junction. The associated vortex flow and the induced pressure in the micro/ nano-channel junction were studied by several authors $^{[7-9]}$. The vortex flows were visualized experimentally, but without the induced pressure distribution being observed in the nanochannel. It is extremely difficult to directly measure the induced pressure distribution in the micro/nano-channel. The present study focuses on the direct observation of this induced pre- 
ssure field, especially, in the nano-channel.

The propagation of the fluorescence in an array of nano-channels was observed in the present experiments. The observation results, as shown in Section 2, indicate that the propagation lengths in each channel of the nano array are different and an analogous parabolic front is established across the entire array. It is demonstrated that the induced pressure distribution at the micro/nano-channel junction drives the fluorescence propagation passing through the nanoarray. In Section 3, two hypotheses are proposed for analyzing the induced pressure based on the Navier-Stokes (N-S) equation and the Nernst-Planck equation, accompanying with the results of a transient 2-D numerical simulation. The last section is the conclusion.

\section{Experimental apparatus and methods}

\subsection{Experimental setup}

The experiments were performed on a fluorescent inverted microscope (Olympus IX71), equipped with a $10 \times / \mathrm{NA}=0.3$ objective lens. An electronmultiplying charge coupling device (EMCCD, DV885) was used to record the images. A voltage source (HV1005) was used to apply the electric potential.

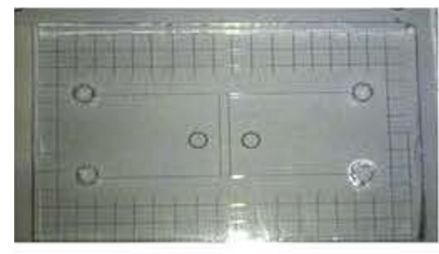

(a)

Fig.1(a) A photo of the glass chip

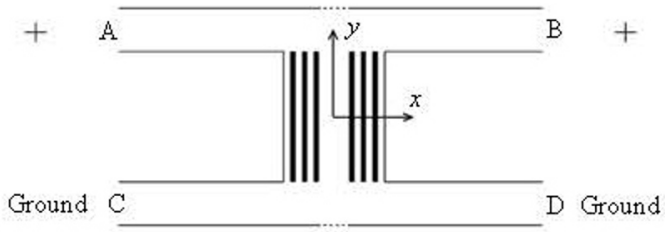

(b)

Fig.1(b) Schematic diagram of the microchannel with a nanochannel array

\subsection{Chip fabrication}

A glass chip employed in the present experiment is shown in Fig.1(a). A schematic diagram of the nano-channel array connected with two micro-channel reservoirs is shown in Fig.1(b), where A-B and C-D are marked. The nominal size of the micro-channel is $40 \mu \mathrm{m} \times 10 \mu \mathrm{m} \times 0.02 \mathrm{~m}$ and that of each nano-channel is $4 \mu \mathrm{m} \times 100 \mathrm{~nm} \times 200 \mu \mathrm{m}$ (width $\times$ depth $\times$ length). The nano-channel bundle consists of 20 channels placed side by side in a linear array with a total width of $120 \mu \mathrm{m}$. The entire glass chip is produced by the
Dalian University of Technology. (see Xu et al. ${ }^{[10]}$ for details).

\subsection{Reagents}

The sodium borate buffer solution was prepared by using Di-water (Millipore company) at a concentration of $0.1 \mathrm{mM}$ and a $\mathrm{pH}$ value of 9.2.

Two portions of this fluid were prepared. The fluorescence Calcein (an analytical reagent) was diluted into one portion of this solution at a mass concentration of $10 \mu \mathrm{M}$ and filled into the A-B micro-channel at the beginning of the experiment. The other portion was filled into the C-D micro-channel. Compared with other experiments ${ }^{[4]}$, in which both microchannels were filled with the fluorescence, in our experiment, only one micro-channel is filled with the fluorescence so that the state of the flow passing through the nano-channel array can be observed.

\subsection{Experimenl procedure}

At the beginning of the experiment, one portion of the sodium borate buffer solution with the fluorescence was filled into the upper micro-channel (A-B) and another portion of the sodium borate buffer solution without the fluorescence was filled into the bottom micro-channel (C-D). Platinum wires were then put into the reservoirs to provide the electric connection. A potential difference was then applied between A-B and C-D micro-channels (see Fig.1(b)) while C-D ends were grounded. The flow fields in the two micro-channels and in the array of nano-channels were observed with the EMCCD at 21 frames/s. The images were captured in sequence with an exposure time of $20 \mathrm{~ms}$ and an interval of $27 \mathrm{~ms}$.

The main experiment was conducted as described above with $50 \mathrm{~V}$ potential drop across the two microchannels. Also, we used the potential drops of $100 \mathrm{~V}$, $300 \mathrm{~V}, 500 \mathrm{~V}$ and a reduced buffer concentration of $0.01 \mathrm{mM}$ in the tests.

\section{Experimental results}

The results of the present experiments are as follows. After an electric potential was applied to the micro-channel A-B, a depletion zone was observed to form in the A-B micro-channel (that is, at the anode of the nano-channel array) at $t=1.24 \mathrm{~s}$ (Fig.2(a)). At $t=3.91 \mathrm{~s}$, the depletion zone continued to extend, while the enrichment zone developing in the C-D micro-channel (the cathode end of the nano-channel array) as shown in Fig.2(b). At this instant, a Fluorescence Concentration Gradient Interface (FCGI) appeared in the nanochannel array. At $t=7.03 \mathrm{~s}$, it was observed that the fluorescence solution was propagating in each nano-channels of the array from the A-B micro-channel towards the C-D micro-channel. 
The position of the FCGI assumes an analogous parabolic form (as shown in Fig.2(c)). At $t=14.86 \mathrm{~s}$, the position of FCGI was near the bottom micro-channel. In addition, the gray scale values of the fluorescence in the nano-channel were always less than those in the depletion area. While in the enrichment area (that is, at the C-D channel end of the nano-channel array), the fluorescent gray values increased with time.

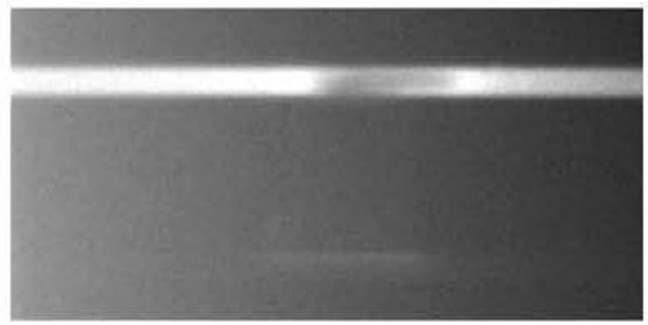

(a) $t=1.24 \mathrm{~s}$

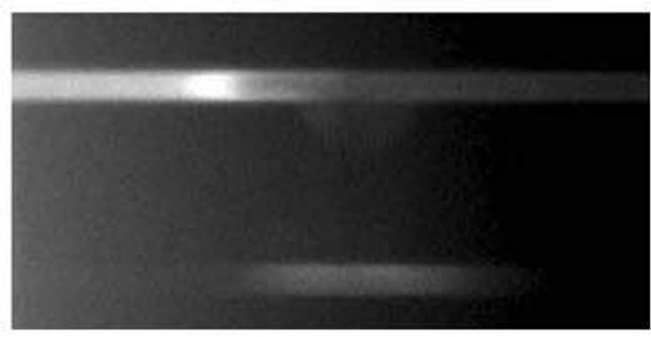

(b) $t=3.91 \mathrm{~s}$

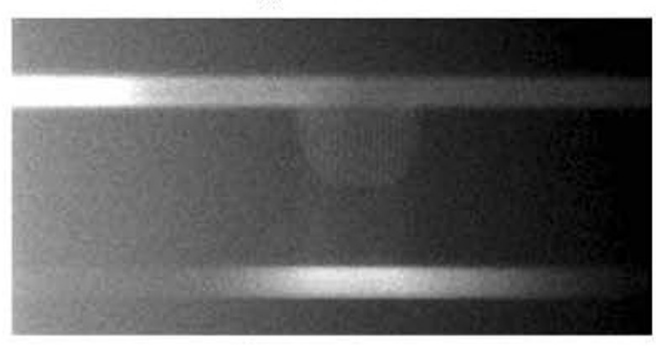

(c) $t=7.03 \mathrm{~s}$

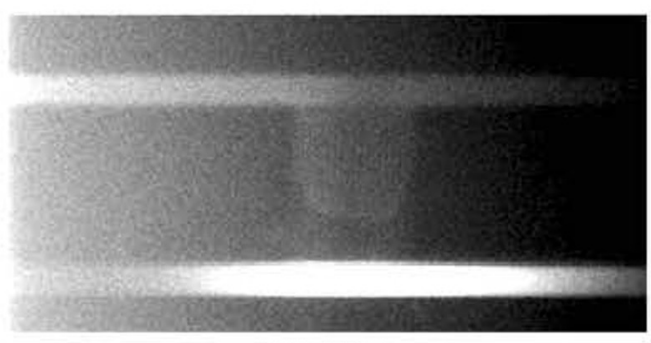

(d) $t=14.86 \mathrm{~s}$

Fig.2 The FCGI evolution in the nanno-channel array with a depth of $100 \mathrm{~nm}$ (the image size is $800 \mu \mathrm{m} \times 400 \mu \mathrm{m}$ )

The front positions of the FCGI in each nanochannel at different moments are shown in Fig.3. They assume an analogous parabolic shape. The average propagating velocity of the FCGI calculated from Fig.3 is shown in Fig.4. The velocity in the center of the nano-channel array is found always higher than the velocities at the edges. For example at $t=3.91 \mathrm{~s}$ the maximum velocity, $v_{\max }$, is about $20 \mu \mathrm{m} / \mathrm{s}$ while the velocities at the edges are only in the range of $0 \mu \mathrm{m} / \mathrm{s}-5 \mu \mathrm{m} / \mathrm{s}$. With the time, the maximum velocity decreases. At $t=7.03 \mathrm{~s}, v_{\max }=15 \mu \mathrm{m} / \mathrm{s}$, and the corresponding velocity values at the edges of the nano-channel array are about $6 \mu \mathrm{m} / \mathrm{s}$. At $t=14.86 \mathrm{~s}$ the maximum velocity is reduced to $9 \mu \mathrm{m} / \mathrm{s}$ while the edge velocities are kept on the level of $5 \mu \mathrm{m} / \mathrm{s}$. The velocity results indicate that the propagation of fluorescence is faster in the middle part than at the edges, however, this propagation becomes much slower as time progresses.

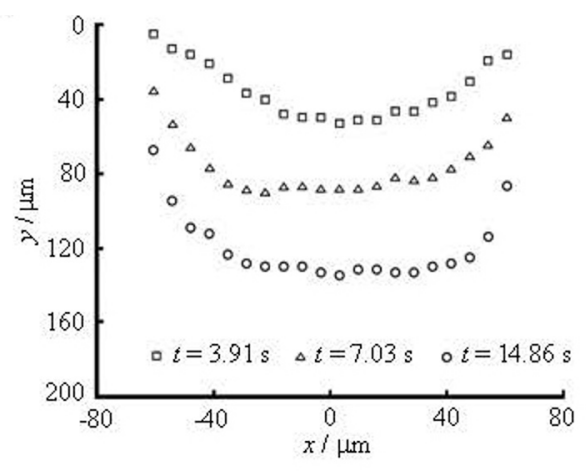

Fig.3 The positions of FCGI's front at different time corresponding to Figs.2(b)-2(d). The location of $x$ axis is at the middle of the nano-channel array

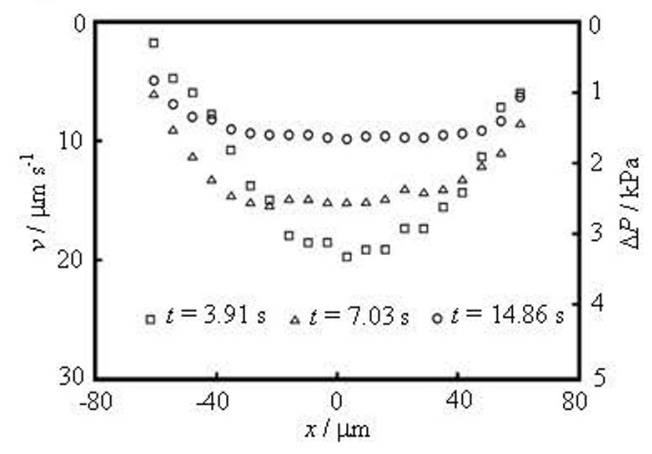

Fig.4 The average velocity of the propagating FCGI and the estimated pressure at different moments

According to the well known Poiseuille formula, the velocity in a channel is proportional to the pressure drop, which can be used to estimate the pressure drop across the nano-channel. The hypothesis involved in the Poiseuille formula will be discussed in Section 3. The Poiseuille formula takes the form

$V=\frac{d^{2} \Delta P}{32 \mu L}$

where $V$ represents the average velocity of the solutions in the nano-channel, and $d$ is the hydraulic 
diameter of the nano-channel. The nano-channel length is $L$ while $\Delta P$ denotes the pressure drop between the two ends of the nano-channel. Using $V$, as shown in Fig.4, we can calculate $\Delta P$ and display it in the same figure. At $t=3.91 \mathrm{~s}$, the pressure in the center of the nano-channel array $\Delta P_{\max }$ is about $3500 \mathrm{~Pa}$. After $3 \mathrm{~s}$, at $t=7.03 \mathrm{~s}, \Delta P_{\max }$ decreases to $2500 \mathrm{~Pa}$. And at $t=14.86 \mathrm{~s}, \Delta P_{\max }$ is further reduced to about $1500 \mathrm{~Pa}$. From Fig.4, it is seen that the center of the experimental $\Delta P_{\max }$ is three times higher than the pressure at the edges (which remains at the level of about $1000 \mathrm{~Pa}$ in the experiment). These estimates provide qualitative information about the pressure distribution along the axis of the micro-channel near the micro/nano junction.

\section{Discussions}

In the section above, we use Poiseuille formula Eq.(1) to calculate $\Delta P$ from the velocity distribution (Fig.3). This estimate is based on two hypotheses, which will be analyzed in this section. Before the analysis, we first discuss the continuity assumption and the validity of the N-S equation for nano-channel flows. For gas, according to the analysis of Bao and $\operatorname{Lin}^{[11,12]}$, when the Knudsen number $K n<0.1$, the continuity assumption is still valid. For liquid (we refer to water here), the mean free path of water molecules is not known due to the fact that water molecules can not move freely like gas, and thus it is difficult to determine $K n$ number. If the interspace of water molecules, about $0.3 \mathrm{~nm}$, is taken as an approximation of the mean free path, for a $100 \mathrm{~nm}$ scale nano-channel, the Knudsen number $\left(\sim 10^{-3}\right)$ still falls in the continuum region. It is estimated that the N-S equation still works down to $1 \mathrm{~nm}$ scale for liquid flow ${ }^{[13]}$. In the present experiment, the hydraulic diameter of the nano-channel is $200 \mathrm{~nm}$, so the continuity assumption and the N-S equation are still valid.

The hypotheses of our analysis will be discussed in two parts:

(1) Ion transportation

Figures. 2(a)-2(d) show that the FCGI is formed as the depletion region is developed at the micro/ nano-channel junction, and extended along the microchannel. The flux of the ions in the nano array is determined from the Nernst-Planck equation

$$
J=-D \nabla c-z F \mu_{e p} c \nabla \Psi+\boldsymbol{V} c
$$

where $c$ denotes the concentration of the species, $J$ represents the flux of that species, $z$ is the valence of the species, $\Psi$ is the electric potential, $\mu_{e p}$ is the ionic mobility of the solution and $F$ is the Faraday constant $\left(9.65 \times 10^{4} \mathrm{C} / \mathrm{mol}\right)$. The first term in the right hand side of this equation represents the flux of the concentration, which is proportional to the gradient of the concentration scaled by the ion diffusion coefficient, $D$. The second term represents the flux due to the electromigration. The last term specifies the flux of the concentration due to the fluid velocity field. The propagation of the fluorescence in the nano array is a result of the electric field, the fluid flow and the ion diffusion. However, in the nano-channel, the characteristic parameters, the diffusion coefficient $D \square$ $O\left(10^{-9} \mathrm{~m}^{2} / \mathrm{s}\right)$, the electric potential $\Psi \square O(10 \mathrm{~V})$, the electric mobility $\mu_{e p} \square O\left(10^{-12} \mathrm{~s} / \mathrm{Kg}\right)$, show that the ratio of the electrical potential over the diffusion $F \mu_{e p} \Psi / D \square O\left(10^{2}\right)$ is large. Therefore, the influence of the diffusion can be neglected, and the velocity of the ions $\boldsymbol{V}_{\text {ion }}$ consists of the electro-migration $\boldsymbol{V}_{e}$ and the fluid velocity $\boldsymbol{V}_{\text {fluid }}$, which can be written as $\boldsymbol{V}_{\text {ion }}=\boldsymbol{V}_{e}+\boldsymbol{V}_{\text {fluid }}{ }^{[14]}$. Because the electrical potential $\Psi$ is applied on the microchannels and the length of microchannel is in the order of centimeters, much longer than the junction of the micro/nano-channel $(\sim 120 \mu \mathrm{m})$, we may adopt the first hypothesis that the electric potential $\Psi$ along $x$ can be considered as uniform in the junction and its gradient $\nabla \Psi$ takes a constant value, as mentioned in the references ${ }^{[15,16]}$. Therefore, the distribution of the velocity driven by the electromigration should assume nearly a flat line. Thus, the electrical term $\boldsymbol{V}_{e}$ could not be responsible for the analogous parabolic velocity distribution in the nano-channel array since the electric potential is nearly uniform along the nano-channel array. $\boldsymbol{V}_{\text {ion }}$ only depends on $\boldsymbol{V}_{\text {fluid }}$, which will be discussed below.

(2) Bulk fluid transportation

The fluid flow is governed by the N-S equation. In the micro/nano-scale, the Reynolds number is low enough, the N-S equation can be simplified as the Stokes equation

$$
\rho \frac{\partial V}{\partial t}=-\nabla P+\mu \nabla^{2} V-\rho_{e} \nabla \Psi
$$

where $\rho_{e} \nabla \Psi$ denotes the electrostatic body force term, $\rho_{e}=F\left(z_{+} c_{+}+z_{-} c_{-}\right)$is the net charge density of ions, $\mu$ denotes the dynamic viscosity of the fluid, $\mu \nabla^{2} V$ represents the viscous term. It is clear that the velocity of the fluid is influenced by the pressure, the electrical field and the viscosity. As stated above, the electro-migration $\boldsymbol{V}_{e}$ is nearly the same in each nanochannel, and has little contribution to the parabolic 
velocity distribution in the nano array. Therefore, the second hypothesis proposed here is that the pressure gradient term, $\Delta P$, plays the dominant role in the formation of the analogous parabolic FCGI.

The estimated pressure is not quite accurate due to the fact that the average velocity value is obtained from experiments, with the effects of the ion diffusion and the electric potential being neglected as just noted. However, these results represent the experimental evidence of the pressure distribution in the micro/nanochannel flows, which has not been noted before. This observation provides some further basic information to understand these complex flows.

A basic numerical model is tested by a simple verification. It can not fully simulate all the experimental details, but it can help us catch the mechanism in the system.

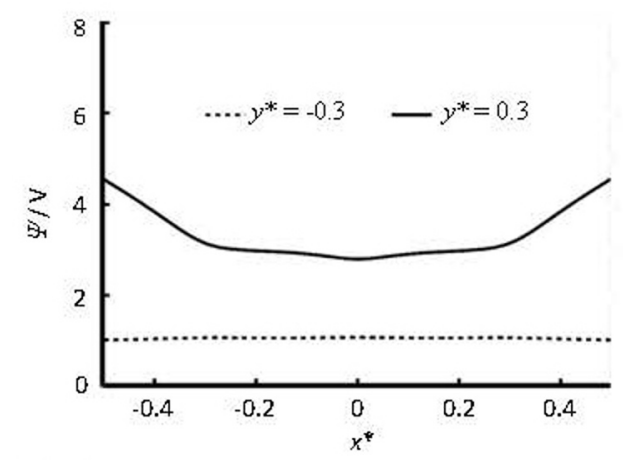

Fig. 5 The calculated pressure distribution along $x^{*}$ axis of the micro-channel in the micro/nano-channel junction. The inset is the computation area of the numerical study, both $x$ and $y$ are normalized by the width of micro-channel

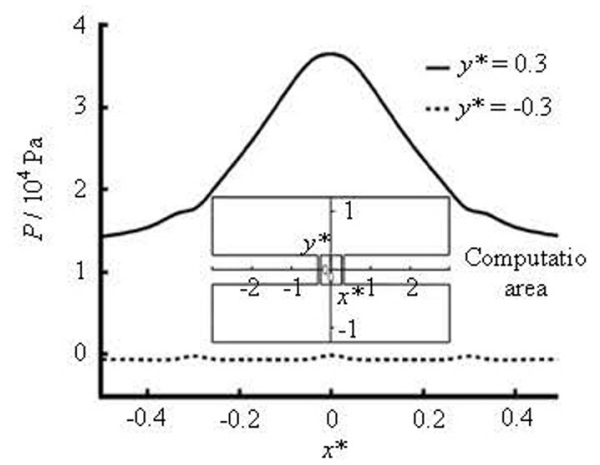

Fig.6 The potential distribution along the $x^{*}$ axis of the microchannel near the micro/nano-channel junction. Both $x$ and $y$ are normalized by the width of micro-channel

In the numerical simulation, three nano-channels are arranged at $x^{*}=-0.3,0$ and 0.3 , respectively, to represent the nano array (see the inset graph in Fig.5). Their inlets are all located at $y^{*}=0.3$, and the outlets are at $y^{*}=-0.3$. The junction of the micro/nanochannel is thus between $x^{*}= \pm 0.3$. Figure 6 shows the computed electrical potential distribution along the $x^{*}$ axis of the micro-channel. The curve of voltages is found to be approximately flat from $x^{*}=-0.3$ to $x^{*}=0.3$, corresponding to the inlet of the nano-channel array at $y^{*}=0.3$. This result confirms the above hypotheses that the $\nabla \Psi$ is nearly constant along $x$ direction. Hence the electric field could not induce the analogous parabolic FCGI observed in the experiments (see Fig.2(a)-2(d)).

Figure 5 shows the pressures along the $x^{*}$ direction of the micro-channel near the micro/nano-channel junction. The pressure distribution in the depletion zone near the junction between $x^{*}= \pm 0.3$ at $y^{*}=0.3$ is in an analogous parabola profile (solid line), and in the enrichment zone at $y^{*}=-0.3$ it is nearly linear (dotted line). This result is in accordance with the experimental pressure distribution discussed above.

These simulations confirm the existence of an induced pressure distribution in the micro/nano-channel junction and this pressure field is responsible for the formation of an analogous parabolic FCGI in the nano-channel array observed in the experiments.

\section{Conclusions}

In this study, the propagation of the fluorescence concentration in a hybrid micro/nano-channel under external electric field was observed. The main results can be summarized as follows:

(1) A FCGI was observed across the nano-channel array (20 nano-channels, each of $4 \mu \mathrm{m} \times 100 \mathrm{~nm} \times$ $200 \mu \mathrm{m}$ (width $\times$ depth $\times$ length). The FCGI front was shown to have an analogous parabolic shape. The average propagation velocity distributions were obtained from images at different moments.

(2) Based on the Nernst-Planck equation, it is shown that the ion diffusion effect can be neglected as compared with the effects of the electromigration and the fluid convection. Two hypotheses are introduced: (a) the electric potential is uniform and thus the potential gradient can be considered as constant in the junction, and (b) in the N-S equation, only the pressure gradient is responsible for this FCGI. Based on these two hypotheses, the distribution of the induced pressure at the micro/nano junction is evaluated.

(3) A basic 2-D numerical model was used to simulate the flow in a hybrid micro-channel with nano array composed of three nano-channels. The numerical results show the similar distribution of the induced pressure in the micro/nano-channel junction. Also from the electrical potential along the $x^{*}$ direction, it is shown that the values at the inlets of the nano-channels are nearly uniform. 


\section{Acknowledgment}

The authors wish to thank Professor Trevor Moulden for fruitful discussions.

\section{References}

[1] WANG Y.-C., STEVENS A. L. and HAN J. Millionfold preconcentration of proteins and peptides by nanofluidic filter[J]. Analytical Chemistry, 2005, 77(14): 4293-4299.

[2] KIM S. M., BURNS M. A. and HASSELBRINK E. F. Electrokinetic protein preconcentration using a simple glass/poly (dimethylsiloxane) microfluidic chip[J]. Analytical Chemistry, 2006, 78(14): 4779-4785.

[3] KIM S. J., KO S. H. and KANG K. H. et al. Direct seawater desalination by ion concentration polarization[J]. Nature Nanotechnology, 2010, 5(4): 297-301.

[4] KIM S., WANG Y.-C. and LEE J. H. et al. Concentration polarization and nonlinear electrokinetic flow near a nanofluidic channel[J]. Physical Review Letters, 2007, 99(4): 044501.

[5] YU Q., SILBER-LI Z. Measurements of the ion-depletion zone evolution in a micro/nano-channel[J]. Microfluid Nanofluid, 2011, 11(5): 623-631.

[6] JIN X., JOSEPH S. and GATIMU E. N. et al. Induced electrokinetic transport in micro-nanofluidic interconnect devices[J]. Langmuir, 2007, 23(26): 13209-13222.

[7] KUO T.-C., CANNON D. M. and SHANNON M. A. et al. Hybrid three-dimensional nanofluidic/microfluidic devices using molecular gates[J]. Sensors and Actuators A: Physical, 2003, 102(3): 223-233.

[8] CHANG H.-C., YOSSIFON G. Understanding electrokinetics at the nanoscale: A perspective[J]. Biomicrofluidics, 2009, 3(1): 12001.
[9] SILBER-LI Z., KONG G. and YU Q. et al. Observation of vorticity generation in micro/nano-channel flows subject to an electric field[C]. Proceedings of the 11th Asian Symposium on Visualization. Niigata, Japan, 2011.

[10] XU Z., WEN J.-K. and LIU C. et al. Research on forming and application of U-form glass micro-nanofluidic chip with long nanochannels[J]. Microfluid Nanofluid, 2009, 7(3): 423-429.

[11] BAO F.-B., LIN J.-Z. Linear stability analysis for various forms of one-dimensional Burnett equations[J]. International Journal of Nonlinear Sciences and Numerical Simulation, 2005, 6(3): 295-303.

[12] BAO F., LIN J. Burnett simulation of gas flow and heat transfer in micro Poiseuille flow[J]. International Journal of Heat and Mass Transfer, 2008, 51(15-16): 4139-4144.

[13] BOCQUET L., CHARLAIX E. Nanofluidics, from bulk to interface[J]. Chemical Society Reviews, 2010, 39(3): 1073-1095.

[14] KIRBY B. Micro- and nanoscale fluid mechanics: Transport in microfluidic devices[M] Cambridge, UK: Cambridge University Press, 2010.

[15] KIM S. J., LI L. D. and HAN J. Amplified electrokinetic response by concentration polarization near nanofluidic channel[J]. Langmuir, 2009, 25(13): 77597765.

[16] ZANGLE T. A., MANI A. and SANTIAGO J. G. On the propagation of concentration polarization from microchannel-nanochannel interfaces. Part II: Numerical and experimental study[J]. Langmuir, 2009, 25(6): 3909-3916. 